\title{
Refractory Mixed Phenotype Acute Leukemia
}

National Cancer Institute

\section{Source}

National Cancer Institute. Refractory Mixed Phenotype Acute Leukemia. NCI Thesaurus.

Code C148430.

Mixed phenotype acute leukemia that does not respond to treatment. 